\title{
SCHRÖDINGER EQUATION VIA WKB
}

\author{
A. M. MARIN ${ }^{1}$ R. D. ORTIZ ${ }^{2}$ J. A. RODRIGUEZ ${ }^{3}$ \\ ${ }^{1}$ Faculty of Exact and Natural Sciences, University of Cartagena, \\ Sede Piedra de Bolivar, Avenue of Consulado, \\ Cartagena de Indias, Bolivar, Colombia \\ ${ }^{2}$ Faculty of Exact and Natural Sciences, University of Cartagena, \\ Sede Piedra de Bolivar, Avenue of Consulado, \\ Cartagena de Indias, Bolivar, Colombia \\ ${ }^{3}$ Morelia Institute of Technology, Morelia, Mexico.
}

Keywords: WKB method; Schrödinger equation.

ABSTRACT. The purpose of this paper is to construct the asymptotic for natural frequencies of the Schrödinger equation using the method of Wentzel-Kramers-Brillouin (WKB).

\section{INTRODUCTION}

It is interesting to study the Schrödinger equation. Therefore, the vibration of Schrödinger equation has been studied extensively and continues to receive attention in the current literature. Schrödinger equation can be found in [3,5]. For Schrödinger problem with a uniform small parameter, one can use the method (WKB) Wentzel - Kramers - Brillouin, also known in the literature as the approximation Liouville - Green. In $[1,2]$, WKB method was used for finding asymptotics at high frequency, this method is to obtain asymptotic series for solutions with a small parameter.

\section{MATHEMATICAL FORMULATION}

In this section we find the approximate solution as a linear combination of two linearly independent solutions. Substituting solution in boundary conditions, a homogeous system of two equations is obtained. This system has non-trivial solutions when the determinant is zero.

\subsection{SCHRÖDINGER EQUATION}

In [4], it is obtained Schrödinger equation

$$
i \frac{\partial y}{\partial t}=-\frac{\partial^{2} y}{\partial x^{2}}+f y,
$$

where $y$ is the wave function and $f \in C_{0}^{\infty}([-l, l])$ It is assumed that $-l \leq x \leq l$. Looking for the solution of the Schrödinger equation (1) in the form

$$
y(x, t)=e^{-i E t} v(x) \text {, }
$$

where $E$ is the energy. We obtain the equation

$$
E v(x)=-v^{\prime \prime}(x)+f(x) v(x) .
$$

The boundary value problem is

$$
\begin{aligned}
& -v^{\prime \prime}(x)+f(x) v(x)=E v(x), \\
& v(-l)=v(l)=0 .
\end{aligned}
$$


The discrete spectrum of the problem (4) constitutes a sequence $\sqrt{E_{n}}$ of real numberstending to infinity when $\mathrm{n} \rightarrow \infty$. In view of above it can be considered $E=\frac{1}{\varepsilon^{2}}$ where $\varepsilon \rightarrow 0$ [1]. Therefore Schrödinger problem (4) becomes

$$
\begin{aligned}
& -v^{\prime \prime}(x)+f(x) v(x)=\frac{1}{\varepsilon^{2}} v(x), \\
& v(-l)=v(l)=0 .
\end{aligned}
$$

In the next section we calculate the secular equation of the problem (5)-(6).

In this section we state and solve Schrödinger equation at high frequency.

We are searching for non-trivial solutions of problem (5)-(6).

The main result of this paper is as follows

Theorem 3.1 The eigenvalues of the problem (5)-(6) are given by $E_{n}=\frac{1}{\varepsilon_{n}^{2}}$ with

$$
\sqrt{E_{n}}=\frac{n \pi}{l}\left(1+O\left(\frac{1}{n}\right)\right), \quad n=0,1, \ldots, \quad n \rightarrow \infty,
$$

where $\varepsilon_{n}$ is the solution of the secular equation (21).

Proof. Given that $E=\frac{1}{\varepsilon^{2}}$ then equation (5) can be transformed into

$$
v(x)=-\varepsilon^{2} v^{\prime \prime}(x)+\varepsilon^{2} f(x) v(x)
$$

Following the traditional WKB method, the analytical solution can be replaced by a power series given by the following

$$
v(x)=A(x, \varepsilon) e^{\frac{i \phi(x)}{\varepsilon}}, \quad \varepsilon \rightarrow 0
$$

where

$$
A(x, \varepsilon)=A_{0}(x)+\varepsilon A_{1}(x)+\varepsilon^{2} A_{2}(x)+\ldots, \quad \varepsilon \rightarrow 0,
$$

with $\phi(x)$ and $\mathrm{A}_{\mathrm{j}}(\mathrm{x}), j=0,1,2, \ldots$ are smooth functions and unknown.

Replacing (8) and each of the derivatives of $\mathrm{v}(\mathrm{x})$ in (7), we have the following expression

$$
\begin{aligned}
& A(x, \varepsilon)=\left[\phi_{x}^{2}(x) A(x, \varepsilon)-2 i \varepsilon \phi_{x}(x) A_{x}(x, \varepsilon)\right. \\
& \left.-i \varepsilon \phi_{x x}(x) A(x, \varepsilon)\right]+O\left(\varepsilon^{2}\right), \quad \varepsilon \rightarrow 0 .
\end{aligned}
$$


Replacing (9) on both sides of (10) we obtain

$$
\begin{aligned}
& A_{0}(x)+\varepsilon A_{1}(x)+\varepsilon^{2} A_{2}(x)+\cdots=\left[\phi _ { x } ^ { 2 } ( x ) \left(A_{0}(x)+\varepsilon A_{1}(x)\right.\right. \\
& \left.+\varepsilon^{2} A_{2}(x)+\cdots\right)-2 i \varepsilon \phi_{x}(x)\left(A_{0 x}(x)+\varepsilon A_{1 x}(x)+\varepsilon^{2} A_{2 x}(x)+\cdots\right) \\
& \left.-i \varepsilon \phi_{x x}(x)\left(A_{0}(x)+\varepsilon A_{1}(x)+\varepsilon^{2} A_{2}(x)+\cdots\right)\right]+O\left(\varepsilon^{2}\right) \\
& =\phi_{x}^{2}(x) A_{0}(x)+\varepsilon\left[\phi_{x}^{2}(x) A_{1}(x)\right. \\
& \left.-2 i \phi_{x}(x) A_{0 x}(x)-i \phi_{x x}(x) A_{0}(x)\right] \\
& +O\left(\varepsilon^{2}\right), \quad \varepsilon \rightarrow 0 .
\end{aligned}
$$

Equating the coefficients of the asymptotic series in $\varepsilon$ and taking corresponding to $\varepsilon^{0}$ in (11) and using that $\mathrm{A}_{0} \neq 0$ as seen in the equation (15), it can be obtained

$$
\varepsilon^{0}: \phi_{x}^{2}(x)=1 \text {. }
$$

From equation (12) and choosing the corresponding equality to $\varepsilon^{l}$ in (11), we obtain

$$
\varepsilon^{1}: 2 \phi_{x}(x) A_{0 x}(x)+\phi_{x x}(x) A_{0}(x)=0 \text {. }
$$

By equating the asymptotic series, more equations are obtained. We consider only the first two ones, because other equations are of order $\mathrm{O}\left(\varepsilon^{2}\right)$. Since equation (12) has two real roots withopposite signs, we obtain

$$
\phi_{k}(x)=(-1)^{k} x, \quad k=1,2 .
$$

From the equation (13) and separating the functions $A_{0}(x), \phi_{x}(x)$ x and integrating on both sides, it follows that

$$
A_{0}(x)=C\left(\phi_{x}(x)\right)^{-\frac{1}{2}},
$$

where $\mathrm{C}$ is a non-zero arbitrary constant. Therefore, differentiating with respect to $\mathrm{x}$ in the equation (14) and substituting (15), function $A_{0}(x)$ can be expressed as follows

$$
A_{0}(x)=C \text {. }
$$

Therefore, replacing (14) and (16) in (8) which is the solution $v(x)$ of (5), we have

$$
\begin{aligned}
& v_{1}(x)=d_{1} \sin \left(\frac{x}{\varepsilon}\right)+O(\varepsilon), \quad \varepsilon \rightarrow 0, \\
& v_{2}(x)=d_{2} \cos \left(\frac{x}{\varepsilon}\right)+O(\varepsilon), \quad \varepsilon \rightarrow 0,
\end{aligned}
$$

and writing the solution (5) as the first term of the linear combination of $v_{1}(x), v_{2}(x)$

$$
v(x)=c_{1} \sin \left(\frac{x}{\varepsilon}\right)+c_{2} \cos \left(\frac{x}{\varepsilon}\right),
$$

$\varepsilon \rightarrow 0$, where $c_{1}, c_{2}$ are constants. It is noted that $\left(\frac{x}{\varepsilon}\right), \cos \left(\frac{x}{\varepsilon}\right)$ are linearly independent. 
From the solution (19) and boundary conditions (6) yield a homogeneous system of two equations for two constants $c_{i}, i=1,2$. This system has nontrivial solutions when

$$
\left|\begin{array}{rr}
-\operatorname{sen}\left(\frac{l}{\varepsilon}\right) & \cos \left(\frac{l}{\varepsilon}\right) \\
\operatorname{sen}\left(\frac{l}{\varepsilon}\right) & \cos \left(\frac{l}{\varepsilon}\right)
\end{array}\right|=0 .
$$

$\sqrt{E_{n}}=\varepsilon_{n}^{-1}$.

$$
\sqrt{E_{n}}=\varepsilon_{n}^{-1}
$$

Therefore if $c_{1} \neq 0, c_{2}=0$

$$
\sin \left(\frac{l}{\varepsilon}\right)=0, \quad \varepsilon \rightarrow 0, \quad \varepsilon=\varepsilon_{n}=\frac{l}{n \pi}
$$

then

$$
\sqrt{E_{n}}=\frac{n \pi}{l}\left(1+O\left(\frac{1}{n}\right)\right), \quad n \rightarrow \infty, n=1, \ldots
$$

From (22) and initial conditions, it follows the eigenfunction is

$$
v_{n}(x)=c_{3} \operatorname{sen}\left(\frac{x}{\varepsilon_{n}}\right) \text {, }
$$

where $c_{3}$ is an arbitrary constant.

\section{ACKNOWLEDGEMENTS.}

The authors express their deep gratitude to CONACYTMéxico, Programa de Mejoramiento del Profesorado (PROMEP)-México and Universidad de Cartagena for financial support

\section{References}

[1] L. P. Akulenko and S. V. Nesterov, High-precision Methods in Eingenvalue Problems and Their Applications, Chapman Hall, 2005.

[2] M. V. Fedoryuk, Asymptotic Analysis. Linear Ordinary Differential Equations, Springer, 1993.

[3] R. A. Serway and J. W. Jewett, Physics for Scientists and Engineers. Thomson-Brooks/Cole, 2003.

[4] A. N. Tijonov and A. A. Samarsky, Ecuaciones de la Fisica Matemática, Editorial Mir-Moscu, 1972.

[5] P. Zhevandrov and A. Merzon, Asymptotics of eigenfunctions in shallow potential wells and related problems. Amer. Math. Soc. Transl. Ser 2. 208(53) (2003), 235-284. 